\title{
Niñas obligadas a parir. Situación en la Argentina, Colombia y Guatemala
}

\section{Milena Páramo Bernal CLADEM/IDAES-UNSAM}

En los últimos años, los países de América Latina han empezado a discutir la situación de las niñas menores de quince años que enfrentan embarazos, partos y maternidades, una realidad que - como tantas otras que afectan a las mujeres - había pasado largamente desapercibida y desatendida. Su relevancia en el debate público actual se puede atribuir a la mayor conciencia adquirida en la región y en el mundo sobre las múltiples e históricas violencias que enfrentan las mujeres de todas las edades, sus causas y consecuencias y la emergencia de una intensa movilización de feministas y mujeres que en cada país demandan el cumplimiento de las obligaciones estatales en materia de igualdad de género, erradicación de todas las formas de violencia, los compromisos sobre población y desarrollo, entre otros asuntos.

Pero ha sido en el marco de la lucha por el aborto que esta penosa realidad ganó centralidad, dado que tanto la violación sexual como el riesgo de vida de la mujer que en estos casos se acreditan, son causales de aborto que algunos pocos países de la región aún no contemplan, ${ }^{1}$ de ahí su reivindicación, o que están reconocidas como prácticas legales en la mayoría de países pero se obstruye su cumplimiento.

La exposición en medios de comunicación de algunos casos de niñas madres también ha ampliado la repercusión de este problema aunque su abordaje, en general, dista de ser el adecuado en tanto revictimiza a las niñas, viola su derecho a la integridad y la obligación de preservar el secreto médico de las historias clínicas. Además, la difusión suele ser utilizada como mecanismo de presión por determinados grupos de interés para imponer cursos de acción distintos al aborto cuando son solicitados por las niñas y sus familias o los recomiendan los profesionales de la salud ante el riesgo inminente de vida y salud.

Algunos casos con amplia repercusión mediática han sido emblemáticos para mostrar las violencias que padecen las niñas madres, el uso interesado de los medios y por el amplio debate social concitado. Según el CLADEM, las violencias aquí implicadas constituyen un contínuum de violaciones de los derechos humanos de las niñas, consistente en al menos i) el abuso y la violación sexual, lo cual viola su libertad sexual, ii) la obligación de llevar a término embarazos que ellas no buscaron ni desearon y iii) que se les niegue, dificulte, demore y obstaculice su derecho a interrumpirlo, convirtiéndolas en madres por obligación. Ya sea por acción o por omisión del Estado se configura lo que la red feminista denomina Embarazos y maternidades infantiles forzadas (Chiarotti, 2016).

1 El Salvador, Honduras, Nicaragua, Jamaica, Haití, República Dominicana, Suriname. 
El caso al que nos referimos es el de Mainumby, ${ }^{2}$ una niña indígena paraguaya que en 2015 con once años, $34 \mathrm{~kg}$ de peso, 1,39 m de altura y con desnutrición y anemia, resultó embarazada producto de sucesivas violaciones por parte de su padrastro. La niña acudió al hospital junto a su madre para buscar atención por un fuerte dolor de estómago que la aquejaba y allí corroboraron que este provenía de una gestación de alto riesgo. Conocido el diagnóstico, la madre de Mainumby solicitó el aborto, pedido que rápidamente activó un debate nacional sobre la (in)conveniencia de la ley paraguaya que permite la práctica únicamente en los casos de "peligro serio" para la vida o salud de la madre, como acreditaba la niña. Para los sectores religiosos y conservadores, la ley debería modificarse para prohibir taxativamente el aborto en cualquier circunstancia, mientras que los grupos de mujeres, de feministas, de derechos humanos y otros, promovían incorporar la violación sexual como causal de despenalización y fijar criterios claros y amplios para la interpretación de lo que significa "peligro serio" a fin de que las mujeres puedan acceder a la práctica. La sociedad se polarizó.

En adelante, el trato recibido por la niña fue cruel: la internaron en un hogar maternal durante casi cuatro meses aislada de su familia, la llevaron presa a la madre acusada del posible encubrimiento de la violación, lo que en contexto fue más un castigo por insistir en un pedido que el propio Ministro de Salud de la época - sin competencia para hacerlo- se encargó de rechazar. La internación de la niña se asemejó a una detención ilegal con el objetivo de obligarla a llevar a término la gestación como en efecto ocurrió. Se le realizó una cesárea dado que su cuerpo pequeño, poco desarrollado y anémico, no permitía otro modo.

Mainumby es hija de una familia pobre como la inmensa mayoría de niñas que son forzadas a continuar con embarazos hasta el nacimiento, en partos en Paraguay y en los demás países de la región. Esto no quiere decir que las niñas de clases sociales con mejor pasar no resulten embarazadas producto de violaciones, la diferencia radica en que sus familias acceden a abortos seguros en el sistema legal o clandestino en el marco de la reserva. Recordemos que la violencia sexual es un problema de salud pública que afecta a la niñez sin distinción de clase o género. El caso de Mainumby fue presentado por CLADEM y Amnistía Internacional ante la Comisión Interamericana de Derechos Humanos (CIDH), institución que otorgó una Medida Cautelar ${ }^{3}$ ante la gravedad, urgencia y amenaza de daño irreparable ampliamente acreditada.

En las páginas siguientes nos interesa presentar un breve balance sobre la incidencia de los embarazos y maternidades infantiles forzadas en tres países de la región de América Latina y el Caribe-ALAC: la Argentina, Colombia y Guatemala, desde 2000 hasta el presente; también poner de relieve elementos del contexto sociocultural que habilita que esta práctica nociva siga afectando a las niñas pese a los desarrollos normativos que sancionan los abusos y violaciones sexuales y la práctica de aborto por causales. Finalmente, señalamos algunas discusiones recientes sobre maternidad infantil y aborto.

Antes de iniciar este abordaje, es preciso hacer una aclaración conceptual. En los estudios sociodemográficos existe un consenso respecto de fijar la etapa de la adolescencia en el grupo etario comprendido entre 10 y 19 años y, en atención a que la amplitud de este rango agrupa a individuos significativamente diferentes en cuanto al desarrollo físico, psicológico, emocional y social, se suele dividir el grupo en dos: subgrupo de adolescencia temprana (10 a 14 años) y adolescencia tardía (15 a 19 años). En general, las políticas públicas nacionales y regionales sobre sexualidad y

2 El nombre Mainumby y los demás citados en este artículo no son verdaderos, corresponden a los usados en los medios para preservar la identidad de las niñas.

3 La medida cautelar instó al país a permitir el curso de acción que la niña y su familia requirieron, en este caso el aborto. En línea en: https://www.oas.org/es/cidh/decisiones/pdf/2015/MC178-15-ES.pdf 
reproducción dirigen sus estrategias al grupo de adolescencia tardía que, por razones evidentes, es el que aporta el mayor número de nacimientos del total de las adolescentes. Esta "no centralidad" de las adolescentes tempranas o niñas madres ha contribuido a invisibilizar su situación.

\section{Nacidos vivos de niñas entre diez y catorce años en la Argentina, Colombia y Guatemala}

El embarazo adolescente es "tema" en América Latina desde que los países suscribieron el Programa de Acción de la Conferencia Internacional de Población y Desarrollo $(\mathrm{CIPD})^{4}$ en los años 90, pero pese a los avances registrados desde entonces en materia de política sexual y reproductiva en los ámbitos de la salud y la educación y la reducción en las tasas de fecundidad total (número de hijos por mujer), en la edad adolescente (10 a 19 años), la región sigue reportando una de las tasas más altas del mundo solo superada por la región del África subsahariana. La tasa mundial de fecundidad se ubica en 46 nacimientos por cada mil adolescentes, mientras en ALAC es de 66,5 nacimientos cada mil adolescentes (OPS-UNFPA, 2018).

Vale decir que la fecundidad adolescente varía según el subgrupo de que se trate (temprana o tardía) y el país. Esto quiere decir que un país puede mostrar reducciones en los embarazos del grupo de 15 a 19 años e incrementos en el de 10 a 14 años a la misma vez, o el registro inverso; como también que la fecundidad de los dos grupos simultáneamente ascienda o descienda. A continuación, observaremos el comportamiento de la Argentina, Colombia y Guatemala para el grupo específico de adolescentes tempranas o niñas madres.

\section{Gráfico 1. Total de nacidos vivos de niñas de 10 a 14 años en Colombia, la Argentina y Guatemala. 2000 a 2017.}

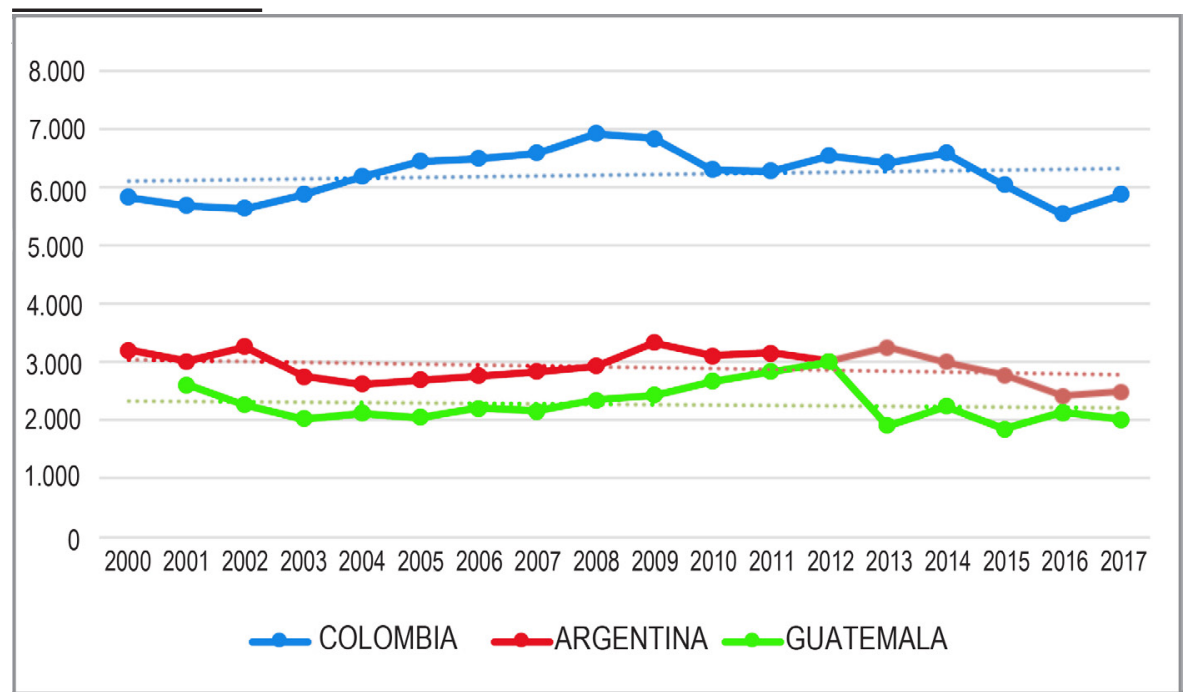

Elaboración propia con datos extraídos de Estadísticas Vitales de Argentina, Colombia y Guatemala. Años 2000 a 2017 - 
Gráfico 2. Tasa de fecundidad específica de niñas de 10 a 14 años. Colombia, la Argentina y Guatemala. 2000 a 2017.

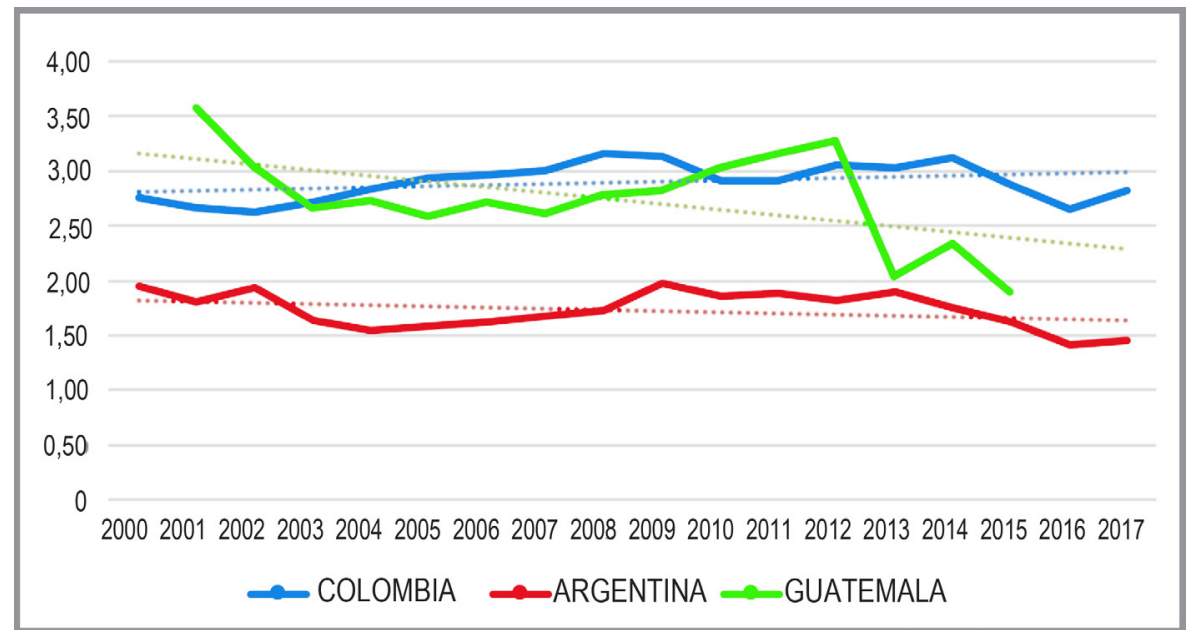

Elaboración propia con datos extraídos de Estadísticas vitales de Argentina, Colombia y Guatemala. Años 2000 a 2017.

El Gráfico 1 muestra la cantidad de nacidos vivos de niñas de entre 10 y 14 años entre 2000 y 2017 en los tres países. El promedio de nacimientos en Colombia en casi veinte años es de 6.207 (esto es 17 nacimientos por día); 2.907 en la Argentina (8 por día) y 2.292 ( 6 por día) en Guatemala. La línea de tendencia de Colombia es levemente ascendente mientras las de la Argentina y Guatemala son levemente descendentes.

Ahora observemos el comportamiento de la tasa de fecundidad específica TFE5 (Gráfico 2). La TFE expresa la frecuencia de ocurrencia de los nacimientos en un grupo de edad específico en un período específico expresado por cada mil niñas. Para nuestro caso, la tasa nos indica cuántas niñas de 10 a 14 años de cada país están expuestas al riesgo de parir en un año. El empleo de la tasa permite comparar más acertadamente la situación entre poblaciones distintas en tamaño, cultura, clases sociales.

La TFE de Guatemala es la que muestra una caída pronunciada en el período. Inicia con 3,59 (casi 4) niñas madres por cada mil niñas de ese grupo quinquenal de edad en 2001 a 1,90 (casi 2) niñas por cada mil en 2015. En Colombia la TFE es 2,68 niñas por cada mil en 2001 y se incrementa a 2,89 en 2015, es decir que en casi veinte años consolida su cercanía a 3 niñas madres por cada mil. Y la Argentina pasa de 1,81 niñas (casi 2) por cada mil en 2001 a 1,63 en 2015. En síntesis: en casi veinte años Guatemala redujo la tasa a la mitad, Colombia no solo no logró reducir su TFE sino que su tendencia es levemente ascendente, y la Argentina la redujo mínimamente. La caída de la tasa de Guatemala se debe también a que su punto de inicio es más elevado que los otros dos países suramericanos.

Las tasas de fecundidad en este grupo podrían parecer poco significativas, sin embargo debe considerarse que lo deseable es que estas sean o tiendan a cero, y que las cifras presentadas en términos absolutos (cantidades) o relativos (tasas) se traduzcan en vidas de niñas que de algún modo se suspenden o se rompen por efecto de violencias que la familia, la sociedad y el Estado no pudieron impedir.

5 La tasa de fecundidad específica es la relación entre el número de nacimientos que ocurren durante un determinado año o período de referencia por cada mil mujeres en edad reproductiva, clasificadas en grupos de edad simples o quinquenales. En este caso se toma el grupo de 10 a 14 años. 
Tal y como quedó ilustrado en el caso de Mainumby, los perpetradores de la violencia sexual son en su mayoría varones de la propia familia o cercanos a la misma. En esta afirmación coinciden distintas investigaciones hechas por país y regionales, incluidas las producidas por organismos del sistema internacional de derechos humanos (Planned Parenthood Global y O'Neil Institute for National and Global Health Law e Ibis Reproductive Health, 2016). La normativa penal de todos los países abreva a esta conclusión al tipificar los delitos sexuales y contemplar agravantes cuando la víctima es menor de edad y, en algunos casos, por incesto. Sobre esto, el Comité de Expertas del Mecanismo de Seguimiento de la Convención Belén de Pará (CEVI) afirma:

La dogmática penal de la región reconoce que los abusos sexuales de niñas menores de 14 años constituyen un delito, dado que se niega validez a su consentimiento. Por ello [...] todo embarazo en una niña de menos de 14 años de edad debe considerarse no consentido y, por tanto, producto de violencia sexual, excepto en los casos donde las relaciones sexuales son entre pares. (MESECVI, 2016)

Ahora bien, ¿qué establecen los códigos penales de estos países sobre la violencia sexual en donde las víctimas son menores? En Colombia, el artículo 208 del Código Penal - $\mathrm{CP}$ - se refiere específicamente al acceso carnal abusivo a persona menor de catorce años y reprime con penas de doce y hasta veinte años de prisión, y contempla en el Art. 211 circunstancias de agravación punitiva cuando se produjere un embarazo, y cuando exista parentesco con la víctima de hasta cuarto grado de consanguinidad, cuarto de afinidad o primero civil. ${ }^{6}$

En Guatemala el Art. 173 del CP tipifica los accesos carnales por cualquier vía contra otra persona y establece una pena que va de ocho a doce años en todos los casos en que la víctima sea menor de catorce años o tenga cualquier discapacidad, aunque no medie violencia física o psicológica. ${ }^{7}$

El Código Penal argentino contempla en su Art. 119 las conductas que atentan contra la integridad sexual de menores: la violación y el abuso sexual calificado (el art. 120 se refiere al abuso sexual que es menos grave que los anteriores). Cualquiera de estas conductas sobre menores de trece años están penadas bajo el supuesto de que por debajo de esta edad cronológica no hay consentimiento válido. Las penas se agravan de ocho a veinte años en caso de que los actos abusivos se cometan contra ascendiente, descendiente, afín en línea recta, hermano, tutor, curador, ministro religioso o encargado de la guarda.

Por su prevalencia, el abuso/violación sexual a niñas, niños y adolescentes en la región ha sido considerado un problema de salud pública y de derechos humanos en América Latina que, a la luz de las cifras de embarazos en niñas, no ha sido disuadido por la amenaza de sanción penal. Los abusos/violaciones a niñas conforman un universo más grande y difícil de estimar que excede por mucho las cifras de aquellas que resultan embarazadas y son obligadas a continuar los embarazos y parir. Podríamos decir que ese grupo representa la punta de un iceberg en cuya base están los casos que no se denuncian (la inmensa mayoría) y los denunciados en los que prima la impunidad.

El agresor viola porque puede. En el caso de las niñas conspira contra ellas su situación de dependencia y vulnerabilidad por ser menores de edad, máxime si el violador es el padre, padrastro, abuelo, hermano o cualquier otro que se aprovecha de la relación de poder. También actúan la amenaza, la vergüenza y la culpa, tanto de ellas como de sus 6 Para consultar el Código Penal de Colombia, ver en línea: https://leyes.co/codigo_penal/211.htm 7 Para consultar el Código Penal de Guatemala, ver en línea:que en los casos de nte. Esta ellan con un e 58 y el proceder institucional marc. o en los actuales tra obstaculizar el pedido http://svet.gob. $\mathrm{gt} /$ temasdetrabajo/lista-de-delitosque en los casos de nte. Esta ellan con un e 58 y el proceder institucional marc. o en los actuales tra obstaculizar el pedido 
madres, que se potencian cuando se depende económicamente del varón violador; las circunstancias familiares en donde los vínculos son problemáticos; la noción cultural de que las/os hijas/os son propiedad de los padres y madres que terminan habilitando prácticas violatorias de los derechos humanos; el desconocimiento de las niñas de temas relativos a la sexualidad y la reproducción que les impide reconocer y registrar las situaciones de abuso y violación como tales. Este desconocimiento es extensible al grupo familiar que, en muchos casos, tampoco cuenta con recursos para distinguir y actuar para proteger a la menor. Estos mismos factores colaboran con la no-denuncia de dichos delitos lo cual termina favoreciendo su comisión así como la extendida impunidad en los casos que sí se denuncian.

Además de la violación sexual, las uniones de hecho o matrimonios en los que una de las participantes es una menor de edad se constituye en causa subyacente de los embarazos infantiles forzados. El sistema internacional de derechos lo considera una práctica cultural nociva propiciatoria de la persistencia de los abusos y el incremento de los embarazos y partos a temprana edad. ${ }^{8}$ Guatemala, dentro de la región de América Latina, es uno de los países en los que matrimonios y uniones de hecho estaban permitidos hasta la modificación del régimen legal en 2015 que fijó en dieciocho años la edad mínima para ser contrayente (con permiso especial también a los dieciséis años). Lo común en estos vínculos en el país centroamericano era la unión entre hombres mayores con mujeres de edades muy tempranas, lo que incidió directamente en el inicio precoz de la vida sexual y reproductiva de las niñas, las altas tasas de fecundidad en los dos grupos de adolescentes como se verifica en el Gráfico 2 -según un estudio de UNFPA-FLACSO (s./f.) el $51 \%$ de quienes embarazan a las niñas de doce años les doblan o triplican la edad-, lo mismo que las altas tasas de mortalidad materna. Se espera que esta medida contribuya a bajar la incidencia de los embarazos no solo entre las adolescentes tempranas sino en el conjunto de las adolescentes.

Las tasas de fecundidad global y adolescente en las zonas rurales de Colombia y Guatemala son habitualmente más altas que las urbanas. El mundo rural está poblado en su mayoría por distintos pueblos indígenas y comunidades afrodescendientes y campesinas que padecen desde hace décadas la intensificación de la pobreza, la exclusión y la violencia en sus territorios.

Esta mayor fecundidad de los pueblos y comunidades se debe a múltiples razones que exceden la pobreza y la falta de acceso a servicios de salud sexual y reproductiva que se da, por ejemplo, en los barrios pobres en las ciudades. Incide aquí la alta valoración de la maternidad como rol fundamental de lo femenino por parte de las comunidades y pueblos, institución que se refuerza y sobrepolitiza en contextos de violencia. Resistir es existir a pesar de la amenaza de extinción humana y cultural que trajeron los actores armados legales e ilegales, y la reproducción biológica es condición necesaria pero no suficiente para este propósito. La reproducción ideológica y cultural complementa el mero hecho de parir y en esta estrategia las mujeres juegan un rol central en tanto madres. Por ello, la "presión" por reproducir la cultura aplica a todas las mujeres con independencia de la edad. Otros factores como el poco estatus de la mujer soltera y sin hijos; las prácticas culturales que habilitan encuentros sexuales con menores; la violencia sexual que sufren las mujeres, niñas inclusive, por varones de su propia comunidad y por otros externos a ellas (p. ej. actores armados legales e ilegales en zonas de conflicto en Colombia) incrementan la fecundidad en las adolescentes.

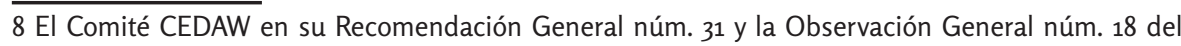
Comité de los Derechos del Niño definen como práctica nociva el matrimonio forzoso, considerado aquel matrimonio en el cual uno de los dos contrayentes es menor de 18 años porque carecen de consentimiento pleno, libre e informado de una de las partes o de ninguna de ellas. 
En la Argentina la mayor cantidad de embarazos en niñas se producen en las provincias más pobladas, pero las tasas más altas se concentran en las zonas rurales de provincias como Chaco, Corrientes y Formosa, históricamente más rezagadas, donde a la población le cuesta acceder a recursos y servicios sobre derechos sexuales y reproductivos. Como se dijo, el abuso sexual es el determinante principal de los embarazos de las menores de edad. ${ }^{?}$

El aborto en los casos de violación están habilitados en Colombia y en la Argentina, no así en Guatemala. En la Argentina durante noventa años el segundo inciso del art. 86 referido a la posibilidad de acceder a un aborto en caso de violación fue objeto de controversias hasta que la medida autosatisfactiva de la Corte Suprema de Justicia de la Nación conocida como Fallo FAL (Fallo C.S.J.N. del 13/03/2012.) resolvió que el supuesto de no punibilidad de la interrupción voluntaria del embarazo aplica para las mujeres con independencia de si esta es capaz o incapaz (idiota o demente). En Colombia el aborto está despenalizado desde 2006 por las mismas causales que en la Argentina: en caso de violencia sexual y peligro para la vida y la salud de las mujeres mediante la Sentencia de la Corte Constitucional C.355/06. Guatemala tiene una ley restrictiva de aborto que lo permite cuando la vida de la mujer corre peligro.

\section{Debates sobre maternidad infantil y aborto}

En los últimos años, la región latinoamericana ha sido el escenario de ingentes concentraciones y movilizaciones convocadas por el movimiento de mujeres y feministas y la Argentina es su epicentro. Cientos de miles se dieron cita desde 2015 en las calles y plazas del país tras la consigna \#NIUNAMENOS en contra de todas las violencias contra las mujeres y conformando la "marea verde" que en 2018 acompañó de principio a fin el debate del proyecto por el aborto legal en el Congreso Nacional. Y cada año, como hace ya más de treinta, miles de mujeres se dan cita en distintas ciudades para vivir esa experiencia inédita de diálogo e intercambio político que es el Encuentro Nacional de Mujeres. En muchos países se dieron movilizaciones réplica frente a preocupaciones compartidas como el feminicidio, las restricciones del aborto, pero también sobre otros tópicos de relevancia como la defensa de la democracia protagonizada por las brasileras ante el golpe orquestado contra la presidenta Dilma Rousseff, la lucha en contra del gobierno represor de Nicaragua que declaró como enemigos a estudiantes y feministas o, también, el protagonismo de las chilenas en la demanda por educación pública y gratuita, entre tantas otras.

Pero también se observa en la región ALAC un brote de la derecha conservadora que ha logrado construir y consolidar articulaciones nacionales, regionales e intersectoriales fuertes y ampliar su poder de convocatoria y movilización especialmente "en contra de". En la retórica de estos grupos hay una evocación constante de instituciones y valores que supuestamente se perdieron y son la causa de las penurias sociales, económicas y políticas que enfrentan los países y la región, por los cuales sienten el llamado a restituirlos. Dentro del balance de pérdidas están la maternidad, la familia, la seguridad, la república, por lo que hay que "Salvar las dos vidas", trabajar para que "con mis hijos no te metas", luchar en contra de "la ideología de género" y extirpar el "marxismo cultural". Una misión moralizadora y redentora.

Muchos políticos han llegado a ocupar cargos de poder político con estas agendas de restauración. Un caso reciente y palmario es Brasil que incluyó en su agenda reaccionaria los tópicos de familia, aborto, homosexualidad, anticomunismo, desde el gobierno

9 http://www.msal.gob.ar/images/stories/bes/graficos/oooooo1079cnt-ninas-dolescentes-menores15-anos-embarazadas.pdf 
golpista de Temer y, más radicalizado aun en el actual gobierno de Bolsonaro. En Colombia, la "defensa de la familia" y la oposición a la denominada "ideología de género" tomó centralidad al momento de obturar los acuerdos de paz entre el gobierno del presidente Juan Manuel Santos y la guerrilla de las FARC, también para poner en jaque la política de educación sexual en los colegios durante ese mismo gobierno. El presidente actual, Iván Duque, representa esos sectores sociales, políticos y religiosos a la derecha del espectro político que están en contra del proceso de paz y de lo que han dado en llamar la "ideología de género".

El presidente de Guatemala, Jimmy Morales, teólogo evangelista y actor cómico, en su candidatura (2015) resaltó su oposición al aborto y al matrimonio gay como valores que encarnaría su eventual gobierno y en eso cumplió. En 2017, tuvo lugar la discusión del proyecto "Ley para la Protección de la Vida y la Familia" conocida como la Iniciativa 52/72 que establecía castigos con penas de prisión a los abortos involuntarios, el agravamiento de las penas a todos los demás, veto al matrimonio igualitario y la prohibición de la educación sexual. La ley estuvo cerca de ser aprobada y habría sido más restrictiva que la del vecino El Salvador que castiga el aborto con prisión de treinta años y más.

En la Argentina, durante la discusión parlamentaria del proyecto IVE, circularon discursos muy radicalizados en contra de la educación sexual, del aborto en casos de violencia sexual, se negó el riesgo de vida en ciertos casos, la magnitud de los abortos clandestinos y su afectación desproporcionada en los sectores más vulnerables de la sociedad. Y emergieron toda suerte de elogios a la maternidad en la niñez. El centenario diario argentino La Nación, que de algún modo representa las voces conservadoras y reaccionarias, en reiteradas oportunidades se ha manifestado en contra del aborto, el matrimonio igualitario, las universidades públicas, el gasto social, también dedicó una de sus editoriales a exaltar la maternidad infantil. "Niñas madres con mayúscula" ${ }^{10}$ fue el titular de la editorial que fue ampliamente rechazada por su apología del abuso sexual y por el uso del eufemístico "instinto maternal" que poseen todas las mujeres. Queda plasmada la voz de ciertos sectores que consideran que las niñas violadas deben parir y punto. Que la violación, el riesgo de vida y salud, que no se haya buscado ni deseado el embarazo o que se las someta a extremo sufrimiento en el proceso de gestación no eximen la obligación de parir. Días después, como si la realidad hubiese querido mostrar lo dramático del tema, se conoció el caso de Lucía.

Lucía ${ }^{11}$ a los diez años fue violada por el novio de su abuela. Vive en Tucumán y un día de febrero del presente año acudió al Hospital del Este buscando alivio a un intenso dolor abdominal. Cursaba un embarazo riesgoso. La madre de Lucía pidió que se le practicara un aborto y la niña dijo al personal de salud en varias oportunidades: "Quiero que me saquen esto que me puso adentro el viejo". El caso se hizo público, su mediatización fue usada para generar polémica alrededor de un pedido que el Código Penal habilita desde 1921, la familia fue hostigada por grupos religiosos y demás organizaciones que enarbolan la bandera de "Salvemos las dos vidas". El Secretario del Sistema de Salud de la provincia intervino para obstaculizar el pedido de aborto, ofreció información falsa ante los medios y médicos sobre la historia clínica de la niña, la aisló en una habitación del hospital a la que solo tenían acceso él, un par de profesionales de la salud contrarios al aborto y el capellán del hospital. Todos loso días, la niña y su madre escuchaban la prédica del capellán sobre la muerte, el pecado y los horrores que envía dios como castigo por el aborto y oían a médicos que les mostraban ecografías, fotos de fetos y las trataban de asesinas. Paralelamente, judicializaron dicho pedido para ganar tiempo de continuidad de la gestación. CLADEM y ANDHES asistieron jurídicamente a la familia de la niña y denunciaron todos los hechos que en suma constituyen trato

10 https://www.lanacion.com.ar/opinion/ninas-madres-con-mayusculas-nid2216199 11 https://www.pagina12.com.ar/176284-la-nina-llora, https://www.perfil.com/noticias/sociedad/elescalofriante-relato-de-las-torturas-que-vivio-lucia-la-nena-de-11-anos-forzada-a-parir.phtml 
cruel e inhumano sobre una menor de diez años. Finalmente, a Lucía le practicaron una cesárea, el nacido vivo murió días después.

En muchos aspectos la historia de Lucía es similar a la de Mainumby. La violación, la situación socioeconómica de la familia, la detección del embarazo pasado el segundo trimestre, que nadie detectara indicios de los abusos sexuales que se cometieron en más de una ocasión, que las niñas desconocieran o no registraran la posibilidad de un embarazo porque no recibieron educación sobre sexualidad. También son similares por la respuesta estatal e institucional. Las niñas se enfrentaron a un dispositivo extralegal que opera desde el sistema público de salud que busca impedir, como en efecto lograron en los dos casos, que las mujeres (niñas en este caso) ejercieran su derecho a abortar en los casos enmarcados en la ley. El dispositivo funciona desde que profesionales de la salud rompen el pacto de silencio médico-paciente y advierten a la prensa y a organizaciones antiderechos sobre un pedido de aborto; aíslan a las niñas, las agobian, les prometen educación, casas, ayuda para el cuidado del bebé, les sugieren la adopción, las amenazan... En algunos casos también recurren al poder judicial para que la gestación gane tiempo y el aborto ya no pueda efectivizarse. En suma, funcionarios/as públicos participan de la tortura de las niñas para obligarlas a continuar y terminar con embarazos producto de violaciones. Mainumby y Lucía tuvieron partos por cesárea, la primera a término y la segunda antes de término. Las dos protagonizaron intentos de suicidio.

Ese imperativo de que las mujeres deben ser madres a cualquier edad, costo, circunstancia y riesgo riñe con lo establecido por la Plataforma de El Cairo sobre el derecho de las mujeres a elegir libremente si quieren ser madres o no. En este sentido, se verifica en la región una suerte de glorificación de la maternidad, el retorno de representaciones tradicionales sobre la triada mujer-maternidad-familia que busca no solo desactivar la potencia de la agenda de las feministas y sus conquistas de las últimas décadas, sino volver a un pasado que se desestructuró en el momento en que las mujeres salieron a trabajar y priorizaron otras expectativas, búsquedas, realizaciones sobre el pretendido omnipresente instinto maternal.

La discursividad de exaltación de la maternidad en estos tiempos cruza fronteras problemáticas como justificar la maternidad en la niñez y excusar la comisión de delitos. Los estudios citados en este artículo y otros son coincidentes en advertir la multiplicidad de afectaciones biopsicosociales en cuerpos y subjetividades que no han madurado física, mental, emocional y socialmente. Los riesgos de ocurrencia de preclampsia, eclampsia, infecciones, fístula obstétrica, mortalidad materna y neonatal se multiplican en las niñas. La salud psicológica, emocional y mental se ve afectada por la presencia de ideas suicidas o intentos, depresión, ansiedad, estrés. Y a nivel sociodemográfico, está verificado que el embarazo y la maternidad las expulsa del sistema educativo o también, que porque no estaban insertas quedaron expuestas a la maternidad. Con baja escolaridad o sin ningún grado escolar, la proyección futura es de reproducción de la excusión y la pobreza.

En medio del debate por aborto legal en la Argentina quedó claramente expuesto que hay un sector de la sociedad que no solo está en contra del aborto legal sino que está en contra de los acuerdos sociales sobre esta cuestión signados hace casi un siglo. Hay aquí un núcleo de discusión que se pretendía cerrado pero no lo está. Si componemos un cuadro de disputas que entronque la cuestión de la maternidad infantil y el aborto a partir de las consignas políticas de los actores en pugna tenemos por un lado, las "madrazas" usando la vindicación del diario argentino La Nación versus las "Niñas No Madres" y la "Maternidad Infantil es tortura"; la "defensa de la vida y la familia" versus "aborto legal y matrimonio igualitario" y la consigna "contra la ideología de género" versus "educación sexual integral". El feminismo se ha hecho cargo de proponer e impulsar los cambios que la reacción conservadora en la región quiere desmontar. 


\section{Q Bibliografía}

"Chiarotti, S. (coord.) (2016). Niñas madres. Embarazo y maternidad infantil forzada en América Latina y el Caribe. Balance regional. Perú, CLADEM. En línea en: https:// cladem.org/wp-content/uploads/sites/96/2018/11/nin\%CC\%83as-madres-balanceregional.pdf

» Federación Latinoamericana de Sociedades de Obstetricia y Ginecología (FLASOG) (2011). Factores relacionados con el embarazo y la maternidad en menores de 15 años en América Latina y El Caribe. Lima, Perú. En línea en: https://www.sguruguay. org/documentos/6factores-relacionados-maternidad-menores-15-anos-lac.pdf

"Fondo de las Naciones Unidas para la Infancia (UNICEF) y Ministerio de Salud de la Nación (2018). Abusos sexuales y embarazo forzado en la niñez y adolescencia. Lineamientos para su abordaje interinstitucional. Buenos Aires. En línea en: $\quad$ https://www.unicef.org/argentina/sites/unicef.org.argentina/files/2018-11/ AbusoSexual\%2BAnexoM\%C3\%A9dico_Digital_Nov2018.pdf

» Fondo de Población de las Naciones Unidas (UNFPA) (2013). Maternidad en la niñez. Enfrentar el desafío del embarazo adolescente. Estado de la población mundial 2013. En línea en: https://www.unfpa.org/sites/default/files/pub-pdf/ES-SWOP2013. pdf

» Fondo de Población de las Naciones Unidas (UNFPA) y Organización Panamericana de la Salud (OPS) (2018). Acelerar el progreso hacia la reducción del embarazo en la adolescencia en América Latinayel Caribe. Informe de consulta técnica. Washington. En línea en: https://www.unicef.org/panama/spanish/EmbarazoAdolescente_ $\mathrm{ESP}(1)$.pdf

»Fondo de Población de las Naciones Unidas (UNFPA) y Facultad Latinoamericana de Ciencias Sociales (FLACSO) (s./f.). Me cambió la vida. Uniones, embarazos y vulneración de derechos en adolescentes. Guatemala. En línea en: https://guatemala. unfpa.org/sites/default/files/pub-pdf/me\%20cambio\%2ola\%2ovida\%20-estudio\%2ouniones\%2otempranas.compressed_o.pdf

"Fondo de Población de las Naciones Unidas (UNFPA) y Ministerio de Salud y Protección Social (2014). Protocolo de atención a la embarazada menor de 15 años. Bogotá. En línea en: https://www.minsalud.gov.co/sites/rid/Lists/BibliotecaDigital/ RIDE/VS/PP/SM-Protocolo-atencion-embarazada-menor-15.pdf

» Mecanismo de Seguimiento de la Convención de Belém do Pará (MESECVI) (2016). Informe hemisférico sobre violencia sexual y embarazo infantil en los Estados Parte de la Convención de Belém do Pará. Washington. En línea en: http://www.oas.org/ es/mesecvi/docs/mesecvi-embarazoinfantil-es.pdf

» OPS-UNFPA (2018). Acelerar el progreso hacia la reducción del embarazo en la adolescencia en América Latina y el Caribe. Informe de consulta técnica.

" Planned Parenthood Global y O'Neil Institute for National and Global Health Law e Ibis Reproductive Health (2016). Vida robadas: un estudio multipaís sobre los efectos en la salud de las maternidades forzadas en niñas de 9-14 años. En línea en: https://ninasnomadres.org/wp-content/uploads/2016/11/PPFA-Stolen-LivesSpanish-april-2016.pdf

》Programa de las Naciones Unidas para el Desarrollo (PNUD) (2017). El embarazo en adolescentes: un desafío multidimensional para generar oportunidades en el ciclo de vida. Diciembre. En línea en: http://hdr.undp.org/sites/default/files/reports/2831/ pnud_do_indh2017web.pd 